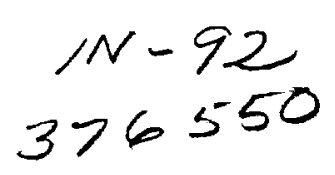

\title{
THE SUN AND THE SOLAR WIND CLOSE TO THE SUN
}

\author{
S. T. Suess
}

N.ASA Marshall Space Flight C'enter, Mail Stop ES82, Huntsville, Alabama 35812. LSA

\begin{abstract}
One of the benefits from the Ulysses, SOHO, and YOHKOH missions has been a strong stimulus to better understand the magnetohydrodynamic processes involved in coronal expansion. Three topics for which this has been especially true are described here. These are: (i) The observed constancy of the radial interplanetary magnetic field strength (as mapped to constant radius). (ii) The geometric spreading of coronal plumes and coronal holes, and the fate of plumes. (iii) The plasma $\beta$ in streamers and the physics of streamer confinement. .
\end{abstract}

\section{INTRODUCTION}

It is said that the structure of the solar corona is imprinted onto the solar wind. This has been demonstrated in many ways, but SOHO and Ulysses are modifying the concept - for both fast and slow solar wind. More specifically, the results are modifying ideas about the physical role of the magnetic field as interpreted using MHD models of coronal expansion.

Consider first fast solar wind, which originates in coronal holes that expand in area by factors of two to tenfold between one and five solar radii. MHD and potential field-based models of the coronal magnetic field make specific, verifiable predictions. Yet, these predictions differ from each other in how geometric expansion varies across a hole and how wind speed depends on the expansion. The differences are still being reconciled, but each prediction must be consistent with the observation that fast wind and the radial magnetic field strength (normalized to constant radius) are surprisingly uniform. What has been added to this is that the solar wind within coronal holes appears to be permeated by filamentary structures in addition to MHD waves. The most well known form of this filamentary structure is the coronal plume. Plumes then apparently becomes thoroughly mixed with the interplume plasma inside 1/3 AU because outside that distance all that is observed is an evolving field of MHD turbulence superimposed on the high speed wind.

Consider next slow wind, which seems to somehow come from inside or from the flanks of streamers and may never achieve a state of steady flow. This puts to rest the old idea of "quiet solar wind" and raises many new questions. There are divergent ideas for the how streamers might be the source of slow wind, but these are being strongly constrained by new data on composition and flow speed, as well as on the properties of streamer cores. It will be important to develop a broader range of streamer models to clarify the main physical processes.

The brief discussion of the above points will concentrate on modeling and the magnetohydrodynamic structure of coronal holes and streamers. The following sources give more information on related topics not dealt with here. First, Ulysses results are summarized in a recent Astronomy \& Astrophysics special issue (v316(2). December (II) 1996). Second, reviews and summaries of recent SOHO results are in the Fifth SOHO Workshop Proceedings (ESA SP-404, "The Corona \& Solar Wind Near Minimum Activity", 1997) and the Solar Physics SOHO Special Issues (v170(1), January \& v175(2), October 1997). In particular, Marsch gives a concise summary of new results and controversies for coronal hole flow in the Fifth SOHO Workshop Proceedings, specifically including the temperatures and temperature anisotropies not discussed here. 


\title{
THE SUN AND THE SOLAR WIND CLOSE TO THE SUN
}

\author{
S. T. Suess
}

NASA Marshall Space Flight Center, Mail Stop ES82, Huntsville, Alabama 35812, USA

\begin{abstract}
One of the benefits from the Ulysses, $\mathrm{SOHO}$, and $\mathrm{YOHKOH}$ missions has been a strong stimulus to better understand the magnetohydrodynamic processes involved in coronal expansion. Three topics for which this has been especially true are described here. These are: (i) The observed constancy of the radial interplanetary magnetic field strength (as mapped to constant radius). (ii) The geometric spreading of coronal plumes and coronal holes, and the fate of plumes. (iii) The plasma $\beta$ in streamers and the physics of streamer confinement. .
\end{abstract}

\section{INTRODUCTION}

It is said that the structure of the solar corona is imprinted onto the solar wind. This has been demonstrated in many ways, but SOHO and Ulysses are modifying the concept - for both fast and slow solar wind. More specifically, the results are modifying ideas about the physical role of the magnetic field as interpreted using MHD models of coronal expansion.

Consider first fast solar wind, which originates in coronal holes that expand in area by factors of two to tenfold between one and five solar radii. MHD and potential field-based models of the coronal magnetic field make specific, verifiable predictions. Yet, these predictions differ from each other in how geometric expansion varies across a hole and how wind speed depends on the expansion. The differences are still being reconciled, but each prediction must be consistent with the observation that fast wind and the radial magnetic field strength (normalized to constant radius) are surprisingly uniform. What has been added to this is that the solar wind within coronal holes appears to be permeated by filamentary structures in addition to MHD waves. The most well known form of this filamentary structure is the coronal plume. Plumes then apparently becomes thoroughly mixed with the interplume plasma inside 1/3 AU because outside that distance all that is observed is an evolving field of MHD turbulence superimposed on the high speed wind.

Consider next slow wind, which seems to somehow come from inside or from the flanks of streamers and may never achieve a state of steady flow. This puts to rest the old idea of "quiet solar wind" and raises many new questions. There are divergent ideas for the how streamers might be the source of slow wind, but these are being strongly constrained by new data on composition and flow speed, as well as on the properties of streamer cores. It will be important to develop a broader range of streamer models to clarify the main physical processes.

The brief discussion of the above points will concentrate on modeling and the magnetohydrodynamic structure of coronal holes and streamers. The following sources give more information on related topics not dealt with here. First, Ulysses results are summarized in a recent Astronomy \& Astrophysics special issue (v316(2), December (II) 1996). Second, reviews and summaries of recent SOHO results are in the Fifth SOHO Workshop Proceedings (ESA SP-404, "The Corona \& Solar Wind Near Minimum Activity", 1997) and the Solar Physics SOHO Special Issues ( $/ 770(1)$, January \& v175(2), October 1997). In particular, Marsch gives a concise summary of new results and controversies for coronal hole flow in the Fifth SOHO Workshop Proceedings, specifically including the temperatures and temperature anisotropies not discussed here. 


\section{GLOBAL CORONAL EXPANSION}

The global solar magnetic field varies in a systematic manner over the sunspot cycle and has well defined properties. In general terms, it is dipolar at solar sunspot minimum and is well approximated by a tilted dipole during the declining phases of the solar cycle. The field becomes more disordered and has much less large scale organization around solar maximum (e.g. Suess, 1993). Global coronal structure reflects this evolution in a logical manner (Billings and Roberts, 1964). The streamer belt follows the magnetic equator in the declining phases and at minimum and this is reflected in the corona appearing elliptic in these phases of the solar cycle, with coronal holes lying over the magnetic poles. The streamer belt becomes distorted around the time of solar maximum when there is no well defined magnetic equator, and the corona appears spiky at small scales and roughly spherical on global scales. Because of the relative simplicity during the declining phases of the cycle and at solar minimum, these times have up until recently been the primary objective of modelers. Global coronal models often still assume a dipole field for the purposes of examining the detailed physics of the numerical results. However, global models designed to compute and predict the detailed morphology of the corona have now gone far beyond the simple axisymmetric dipole case.

Today, daily measurements of the line of sight (LOS) photospheric magnetic field are routinely used as a boundary condition for global coronal models that produce a completely acceptable prediction for the visual appearance of the corona. An example of this is shown in Figure 1, where a 3D MHD model (Mikic \& Linker, 1996) has been used to predict the appearance of the corona during the 24 October 1995 solar eclipse. The prediction is shown on the left and is remarkably like the white light corona shown in the photograph on the right. In a more detailed analysis of several models, their predictions, and related observations, data from Ulysses during the fast latitude scan was compared with predictions from "potential field / source surface" (PFSS) coronal models and from the Mikic \& Linker MHD model. The comparison was between the observed and predicted locations of the heliospheric current sheet (HCS). To do this, the in situ magnetic field was mapped back to the Sun using the constant velocity approximation (Neugebauer et al. 1998). Although none of the models produced a perfect prediction for the location of the HCS, all of them produced an acceptable prediction.

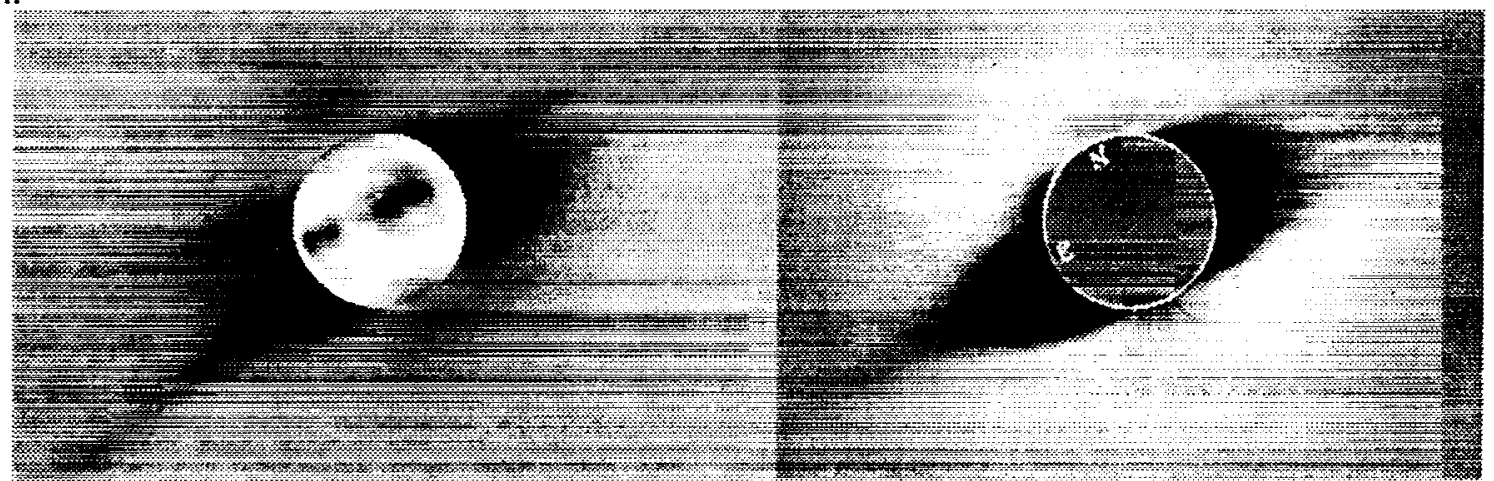

Figure 1. Left: 3D MHD model prediction for the appearance of the corona during the 24 October 1995 total solar eclipse (Jon Linker \& Zoran Mikic, Applied Physics Operation, Science Applications International Corporation, San Diego). Right: Image of the corona taken during the 1995 eclipse (F. Diego, University College, London \& S. Koutchmy, Institut d'Astrophysique, Paris, CNRS). Both images are printed here in negative.

The purpose of PFSS models was originally to simply and quickly predict the location of streamers, of coronal holes, and of the HCS - a task they now are understood to be well qualified for (Neugebauer et al., 1998). Given that the global morphology of the corona can be reasonably well simulated, a discussion of the physical state of the corona is the objective here and this can be accomplished only through combining MHD (not PFSS) models like that shown in Figure I with empirical data because it is presently impossible to directly measure the coronal magnetic field. This has become a very active area of research using data from Ulysses, YOHKOH, SOHO, and interplanetary scintillation (IPS) observations. Figure 2 focuses on this point, depicting Ulysses collecting plasma coming from the parts of the corona remotely analyzed by SOHO. The purpose of this figure is to call attention to one of the most important parameters describing the physical state of the corona - the plasma $\beta$ (the ratio of the thermal to magnetic pressure). As shown, $\beta<<1$ out to at least 10 solar radii $\left(R_{S}\right)$ in coronal holes, while $\beta>1$ in streamers above $\sim 1.2 R_{S}$ (heliocentric). This has specific implications, but first it is necessary to briefly describe how these estimates of $\beta$ were made. 
$\beta$ in coronal holes can be empirically estimated by extrapolating interplanetary observations back to the Sun. For example, $\beta$ (normalized to I AU) was measured at Ulysses to be 3-4 (Suess et al, 1996). Assuming the solar wind temperature varies as $r^{-1}-r^{-0.5}, \beta$ decreases with decreasing heliocentric distance (radius), reaches $O\left[10^{-1}\right]$ at $10 R_{S}$, and reaches $O\left[10^{-2}\right]$ at $5 R_{S}$. Alternatively it is possible to make an empirical estimate by extrapolating upward from the base of the corona. At the top of the transition region $(\sim 7,000 \mathrm{~km}$ above the photosphere), the magnetic field, temperature, and density are $\mathrm{B}=\mathrm{O}[5-10]$ Gauss (DeForest et al. 1998), $T=O\left[10^{6}\right] \mathrm{K}$, and $n_{0}=O\left[10^{7}-10^{8}\right] \mathrm{cm}^{-3}$ (Ahmad \& Withbroe, 1977) Therefore, $\beta=3 \times 10^{-2}$ to $7 \times 10^{-4}$ below coronal holes. Extrapolating upward, using the geometry either from coronal hole models or as derived empirically from coronal hole boundaries, gives $\beta \leq 10^{-2}$ at $5 R_{S}$ (Suess $e t a l, 1998$ ), the same as the inward extrapolation. Coronal hole MHD models are dependent on boundary conditions and physical assumptions and early models did not have as low $\beta$ as determined empirically (e.g. Steinolfson et al, 1982). Those same models also had low flow speed and high density in the open field region and therefore did not really simulate coronal holes. Recent models (e.g. Suess et al, 1996; Wang et al. 1998) have repaired this deficiency and now produce high speed wind from, and have low $\beta$ in, the coronal holes.

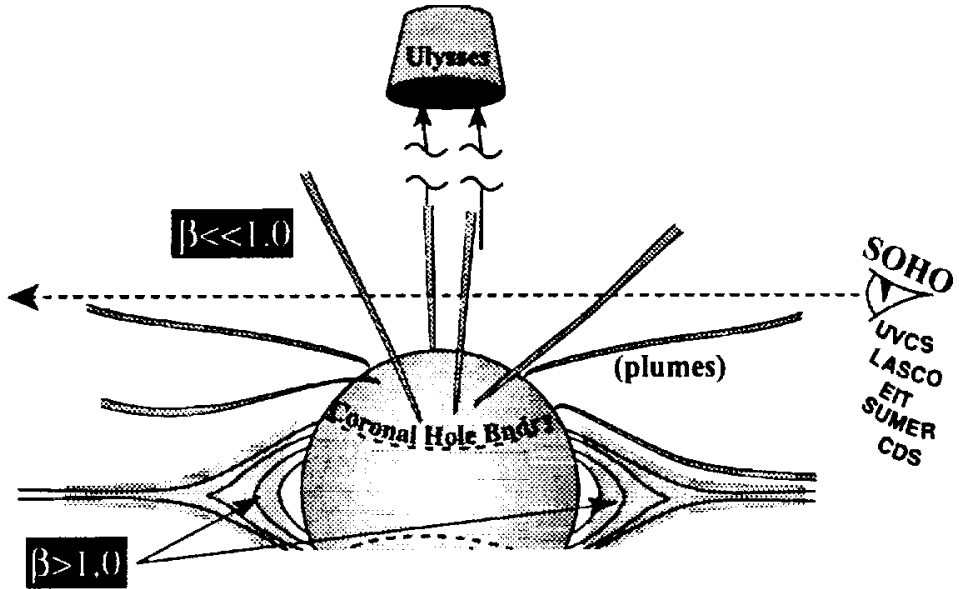

Figure 2. MHD model and empirical results show that $\beta<<1$ in coronal holes to $\sim 10 R_{s}$ and $\beta>1$ in streamers above $\sim 1.2 R_{s}$. The small $\beta$ in holes allows plumes to persist to at least $10 R_{s}$. This cartoon also shows that at certain times Ulysses is able to sample in situ the same plasma that is remotely analyzed by SOHO.
Estimates of $\beta$ in streamers derive both from MHD models of the global corona and from empirical estimates of temperature and density in streamers in combination with potential or phenomenological magnetic field models. MHD models have always given $\beta>1$ from low in streamers out into the solar wind (Steinolfson et al. 1982). This is even true of the original model of Pneuman and Kopp (1972), which predicted $\beta$ to reach $\sim 15$ at $1.6 \mathrm{R}_{\mathrm{S}}$ (Gary \& Alexander, 1998). MHD models with volumetric heating (e.g. Suess et al. 1996) sometimes result in astronomical values for $\beta$, but these may be artificial in the absence of a full understanding of the relationship between heating and momentum addition in coronal expansion. Determining $\beta$ empirically has been a much more difficult task. However, this has now been achieved by combining observations from SOHO/UVCS and YOHKOH/SXT with a PFSS model magnetic field to show that $\beta>1$ above $\sim 1.2 R_{\mathrm{S}}$ in one specific, but apparently typical streamer observed near solar minimum (Li et al. 1998).

\section{CORONAL HOLES}

The result that $\beta<<1$ in coronal holes provides the basis for understanding: (i) Why the radial interplanetary magnetic field (IMF) in the high speed wind is (as mapped to constant radius) independent of latitude. (ii) What the relative and absolute geometric spreading is in coronal plumes and coronal holes. (iii) What the probable fate of plumes is. These three points are discussed here in this order.

\section{The Geometric Spreading of Coronal Holes}

Ulysses is the first mission able to give a snapshot of the variation of the radial IMF, $B_{r}$, with latitude in the high speed wind. Expectations based on PFSS models were that $B_{r}$ would increase towards high latitude (Wang, 1993). The reason for this is that PFSS models have greater geometric divergence at the edges than at the center of open field regions. The photospheric field outside active regions is only weakly dependent on position so the excess divergence at the edges of coronal holes leads to a weaker magnetic field there. High speed flow originates throughout coronal holes and hence it was expected that near solar minimum the field would be weaker in the low latitude high speed wind. 
What was observed is shown in Figure 3, where it is seen that the field strength in the high speed wind is independent of latitude. The explanation for this is that the variation predicted by PFSS models disappears between $\sim I .5 R_{S}$ and $5 R_{S}$. Figure 4 shows this happening in an MHD model (Wang et al, 1995), where the field outside the streamer/plasma sheet depends strongly on polar angle (colatitude, $\theta$ ) at $1.47 R_{S}$, weakly on $\theta$ at $2.47 R_{s}$, and not at all on $\theta$ at $4.88 R_{s}$. Physically, the field gradient across a coronal hole represents a transverse magnetic pressure gradient which cannot be balanced by the thermal pressure gradient since $\beta<<1$. The magnetic pressure gradient is a force directed towards the edges of the hole, onto the flanks of streamers, and which relaxes at the fast mode speed of $-2000 \mathrm{~km} / \mathrm{s}$. Since the solar wind expansion speed is generally much less than $2000 \mathrm{~km} / \mathrm{s}$ at these heights, the gradient completely relaxes in a time short compared to the transit time of the solar wind from the base of the coronal hole to a few solar radii (Suess \& Smith, 1996). The consequence is that by $5 R_{S}$ the radial magnetic field strength is essentially independent of position across a coronal hole. This simple physical argument is fully supported by numerical (Wang et al. 1995) and analytic (Suess et al, 1977) solutions and by physical estimates (Suess \& Smith, 1996).

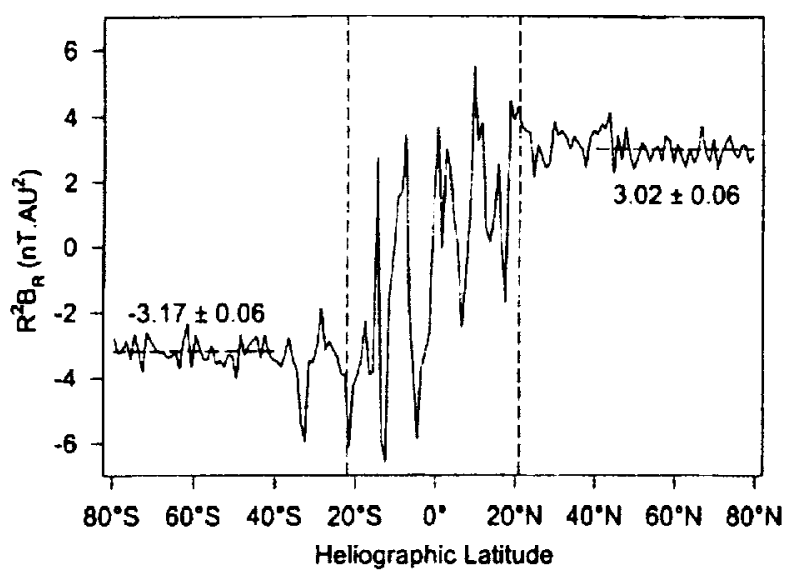

Figure 3. $\mathrm{B}_{\mathrm{r}}$ in the IMF, normalized to $1 \mathrm{AU}$, for Ulysses data collected during the fast latitude scan (summer'94summer'95). The dashed lines delineate the streamer belt (Forsyth et al, 1996). being concentrated at a specific height are not supported by this result since, as noted above, the solar wind speed is, to $O[1]$, independent of position across an individual coronal hole (Phillips et al, 1995).
This result has important implications regarding the geometric spreading in coronal holes. In computations such as that by Wang (1993), it was presumed that the coronal hole magnetic field was nearly a potential field up to heights of $\sim 2.5 R_{S}$. This is now seen to not be the case. Volume currents are important throughout coronal holes and the geometric spreading as a function of position within coronal holes is poorly described in PFSS models except below $1.5 R_{\mathrm{S}}$. Instead, it can be said that the total geometric spreading along streamlines/field lines is nearly the same everywhere in a coronal hole between $1.5 R_{S}$ and $5 R_{S}$. However, the distribution of spreading along individual streamlines will be different being concentrated towards the base of a coronal at the center and at larger radii towards the edge of coronal holes. This means that geometric spreading of coronal hole determined empirically by from the boundary spreading can be assumed to be approximately valid for each individual streamline across the coronal hole. Solar wind models which critically depend on the spreading

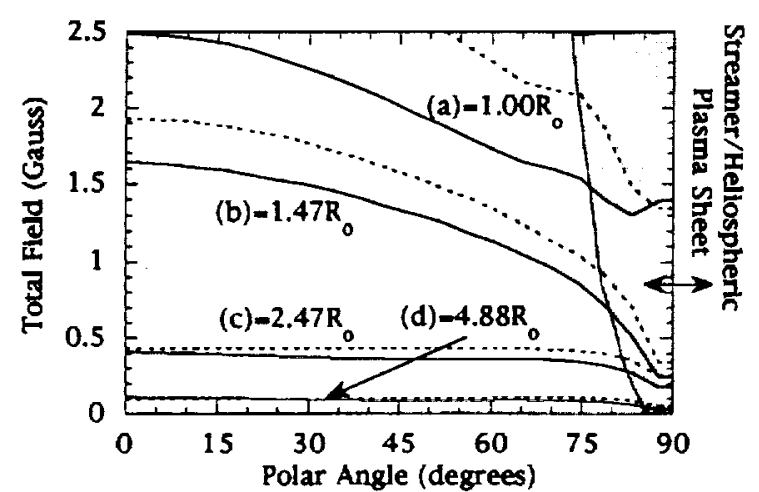

Figure 4. The total magnetic field strength (solid lines), at the indicated radii versus polar angle from the global MHD coronal model of Wang et al. (1995).
Plumes are bright quasi-radial rays observed between one and several $R_{S}$ in coronal holes. They are visible during eclipses and, e.g., from SOHO and SPARTAN-201. After several years of controversy, DeForest et al (1998), by way of a comprehensive set of SOHO observations, have finally shown all plumes lie over photospheric magnetic flux concentrations, although not all flux concentrations have plumes. Being bright, plumes are denser than interplume plasma. In addition, SOHO/UVCS has been used to show that plumes flow at $\sim 150 \mathrm{~km} / \mathrm{s}$ at $2.0 \mathrm{R}_{\mathrm{S}}$ (Corti et al., 1997).

The study of plume dynamics is made both simpler and more difficult when applying the knowledge that $\beta<<1$ in coronal holes. The former because it means plume geometry can be computed separately from plume dynamics inside $10 R_{S}$, the latter because it means that plume and interplume flows are probably strongly mixed somewhere between $10 \mathrm{R}_{\mathrm{S}}$ and $0.3 \mathrm{AU}$. 
Regarding the geometric spreading of plumes, $\beta<<1$ immediately shows that the presence of the plume has no significant effect on the magnetic field geometry (the kinetic energy density is also small compared to the magnetic field energy density). This is a stable situation inside $10 \mathrm{R}_{\mathrm{S}}$, and what happens beyond that distance is addressed below. Because the plasma can be ignored, the computation of the geometry can be divided into two simple calculations: (i) A potential field calculation below $\sim 1.1 R_{S}$ that shows how the field smoothes itself out above the photospheric magnetic flux concentrations. (ii) The global spreading of the surrounding coronal hole computed as described above, either empirically or from a global MHD model. The coronal hole and plume relative spreading are the same above $-1 . I R_{S}$ so this will provide all the information that is needed.

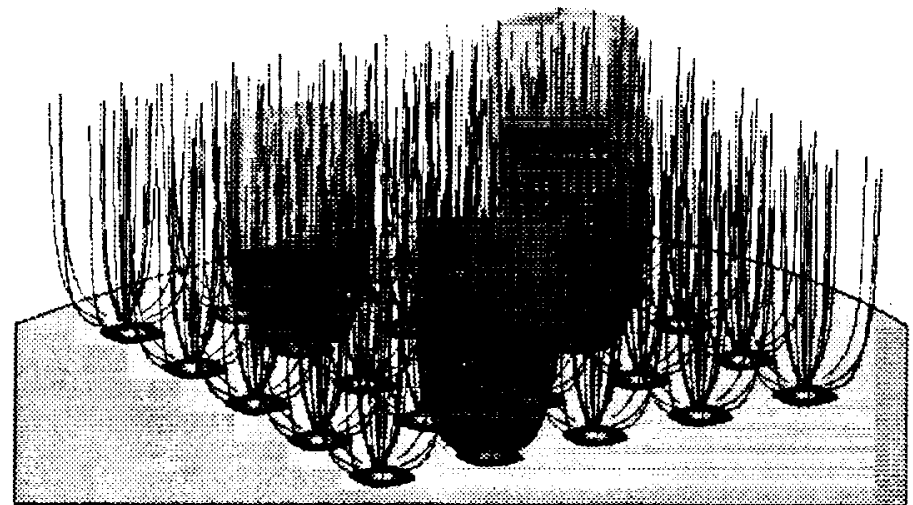

Figure 5. Array of flux concentrations at the photosphere, with rapidly spreading field lines above the photosphere shown to a height twice their typical separation distance $(70,000 \mathrm{~km})$. Some concentrations have plumes, but not all. At $70,000 \mathrm{~km}$, the field has become locally smooth (Suess, 1998).
Figure 5 shows field lines in the low corona, over a field of magnetic flux concentrations. These field lines were computed using the potential field model described by Suess et al (1998). That calculation takes into account all the flux concentrations, even though it is unknown what distinguishes those which are overlain by plumes. The concentrations lie in the chromospheric network, which means they have typical separation distances of $-35,000 \mathrm{~km}$. As is characteristic of potential fields, the overlying magnetic field then spreads out and is approximately uniform by a height comparable to the plume separation distance. Therefore, it can be conservatively stated that the magnetic field in coronal holes is smooth by a height of $70,000 \mathrm{~km}$, or $1.1 R_{s}$.

The "basal plume spreading" shown in Figure 5 can be described by a local spreading factor $f_{l}(r)$. Above $1.1 R_{S}$, the field is smooth and the relative changes in the geometric spreading are essentially identical between

(a)

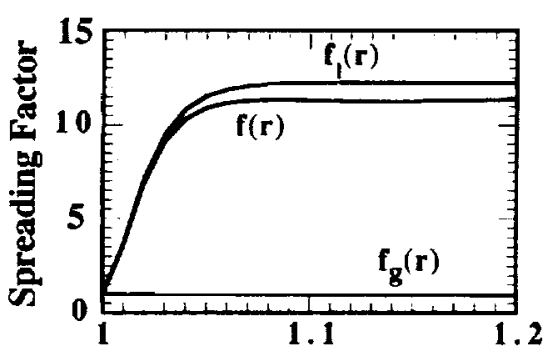

(b)

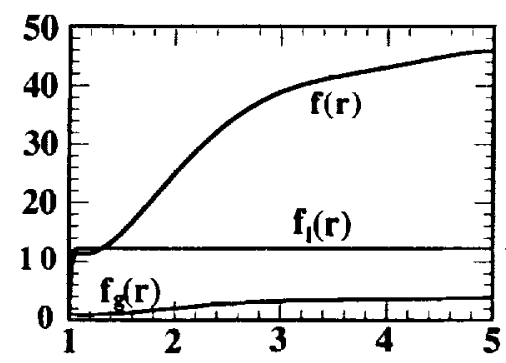

Solar Radii

Figure 6. Combined local and global spreading factors (see text) for a small flux concentration with a $5 \%$ background field. The global spreading is computed along the symmetry axis in the Steinolfson, Suess, \& Wu (1982) model.

in turn, similar to empirical estimates. The global spreading occurs ging spreading can range between 2 and 10 - sometimes being even larger for small coronal holes.

\section{The Fate of Plumes}

As noted, SOHO/UVCS has been used to show that plumes flow at $\sim 150 \mathrm{~km} / \mathrm{s}$ at $2 R_{S}$ (Corti et al, 1997). Plume densities are well enough known as a function of height (e.g. Habbal et al., 1995) to use with typical geometric spreading factors and deduce that the plume flow speed at $5.5 R_{S}$ is $150-230 \mathrm{~km} / \mathrm{s}$. This can be compared with the apparent mean flow speed at that height of $750 \mathrm{~km} / \mathrm{s}$ (Grall et al, 1996) to suggest that interplume plasma is flowing at least twice as fast as plume plasma. This presents a problem because Ulysses detected only high speed solar wind coming from the polar coronal holes, with a variance of only $5-10 \%$. Plumes should stand out clearly if there were large velocity differences. In fact, concerted searches have been made for plume signatures in any plasma parameter (McComas et al, 1995; Poletto et al, 1996), with only weak indication of 
a detection at best. It is possible that plumes simply undergo delayed acceleration, but it would be more than a coincidence it all plumes were to arrive at I AU with just the right flow speed, temperature, and density so as to be indistinguishable from the surrounding fast wind. This is even less likely when it is realized that plumes come with a continuous spectrum of properties rather than, e.g., one specific density contrast. Therefore, it is most likely that plumes are strongly mixed with interplume plasma somewhere between $10 R_{S}$ and $0.3 A U$ where Helios data showed an absence of a strong plume signature.

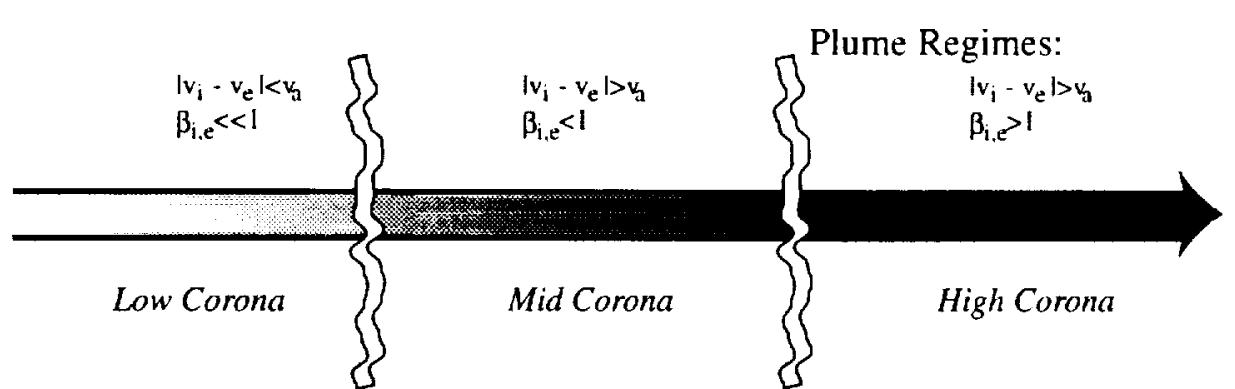

Figure 7. The different regimes encountered by a plume in the corona. $v_{a}$ is the Alfvén speed and ' $i$ ' and ' $e$ ' subscripts referenceproperties internal or external to the plume. Mid corona is somewhere around $10 \mathrm{R}_{S}$ (Suess, 1998). $\mathrm{km} / \mathrm{s}$. However, the Alfvén speed decreases with height, eventually reaching only $\sim 50 \mathrm{~km} / \mathrm{s}$ at $1 \mathrm{AU}$, and the shear speed thus exceeds the Alfven speed somewhere in the outer corona. When this happens, the shear interface has been shown to become unstable and disrupts the interface, producing large amplitude Alfvénic fluctuations (Hardee and Clarke, 1995).

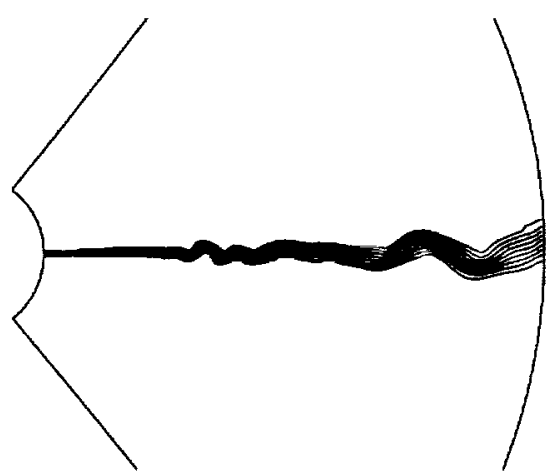

Figure 8. Magnetic field lines for a jet moving into an expanding external atmosphere in cylindrical geometry (Hardee \& Clarke, 1995). The jet is magnetized and remains stable until it becomes superAlfvénic. It then destabilizes abruptly. There is no external field.

" doomed to disruption". This is a very robust result because it fully verifies the stability arguments based on the dispersion relation for linear waves. Nevertheless, the study of plume instability and mixing with interplume plasma is in its very early stages and much work needs to be done to understand what role, if any, the $\mathrm{KH}$ instability has in mixing plumes with interplume plasma and producing the MHD fluctuations commonly detected in the interplanetary medium.

\section{STREAMERS}

Interest in the physical processes in streamers has always been high because they are the location where coronal mass ejections (CMEs) occur. This interest has increased over the past few years with growing questions about the origin of the slow solar wind and the discovery of very small transient releases from streamers even at solar minimum (Sheeley et al, 1997). 
It has long been known that slow wind somehow originates from the streamer belt (Feldman et al, 1981: Gosling et al, 1981) and ideas for how this worked have generally been based in steady state flow models in which it is hypothesized that the spreading functions along streamlines producing slow wind are in some way significantly different than for fast wind. This concept is still tenable (Noci et al, 1997), but alternative hypotheses are also being developed with growing evidence that the slow solar wind source is inherently time dependent. One such piece of evidence comes from SOHO/UVCS, where was found that there are compositional differences inside streamers, with the flanks being less fractionated that the cores (Raymond et al, 1997). The lower fractionation on the flanks suggested a period of gravitational settling followed by a transient outflow. Another piece of evidence is the discovery of continual small sporadic releases of mass from streamers even at solar minimum. This was accomplished with the SOHO/LASCOC2 coronagraph (Sheeley et al, 1997). The releases begin near the tops of streamers, are not visible low in streamers, and they move with speeds like those predicted in slow solar wind steady flow models - although they are inherently a transient flow. In response to these discoveries, several ideas have been put forward for why slow solar wind might originate in transient releases from streamers (Schwadron et al, 1998; Suess et al, 1996; Dahlburg et al, 1998). To evaluate these proposals, it is important to understand the physical conditions in streamers and it is this which is addressed here.

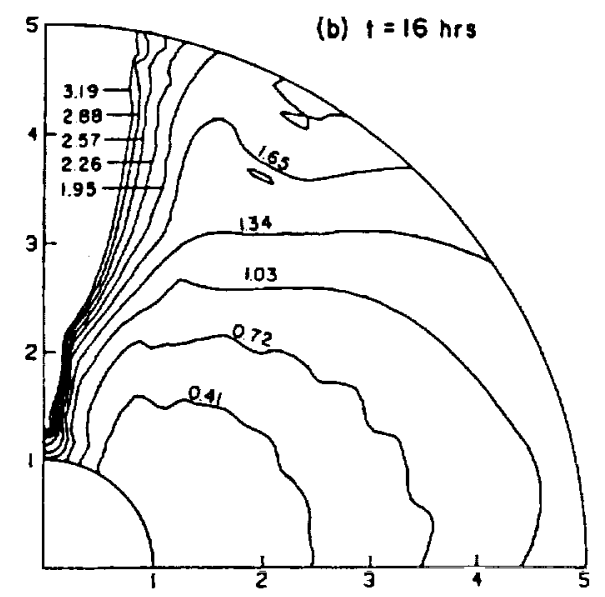

Figure 9. Plasma $\beta$ from the MHD global coronal model of Steinolfson et al (1982) in the case $\beta=0.5$ at the base of the streamer (top of figure).
The point made with Figure 2 regarding streamers is that throughout most of their volume, $\beta>1$. This is reiterated in Figure 9 which shows a contour plot of $\beta$ for the Steinolfson et al (1982) model for a polytropic with $\gamma=1.05$. The model in Figure 9 is for ideal MHD and is typical of the results obtained from such models. But, streamers have long been described as "magnetically confined", so this poses the question: "what is holding streamers down?" The answer can be given in terms of the above description of the magnetic field in coronal holes expanding towards the sides of the holes and pressing on the flanks of streamers. To $O[1]$, streamers are contained by the strong magnetic fields in coronal holes exerting force on the sides of the streamers. The principal effect of the closed field lines near the tops of streamers is to keep them from continuously leaking plasma. This could be contrasted with a simple magnetic pinch which has no such closed lines of force at the ends of the pinch and therefore always leaks plasma. It is only near the base of streamers, below $1.2 \mathrm{R}_{\mathrm{S}}$, where the field can be approximated as a potential or force-free field and the curvature forces are important for containing the plasma. Thus, streamers are magnetically confined, but the physics of this confinement does not necessarily follow the intuitive idea that the curvature forces in the magnetic loops of the streamer are primary source of the confining force.

With this understanding of the physics of streamer confinement and that $\beta>1$ in streamers, it becomes more important to analyze the heating in streamers since the thermal pressure is likely to have a major effect. This has already been discussed in two studies. The first was by Steinolfson (1988) who showed that heating in streamers leads to simulated CMEs that better reproduce observations. In another study, Wang et al. (1995) compared simulated CMEs with and without volumetric heating to show that there O[1] differences between the two cases. Since it is commonly suspected that streamers are heated, which is supported by empirical results show streamers are essentially isothermal ( $\mathrm{Li}$ et al, 1998), the heating of streamers becomes an important modeling problem.

Under conditions of ideal MHD and no heating, steady solutions for streamer structure have long been known to exist (Pneuman \& Kopp, 1972; Steinolfson et al, 1982). But, when volumetric heating is added, there is a qualitative change. In a polytropic gas, there are only two ways energy can be added - by increasing either the kinetic energy or pressure. In the ideal streamer, the flow speed is zero and hence only the pressure can change, increasing with time as heat is added. As the pressure (and $\beta$ ) increases, the top of the streamer inflates and eventually opens to release solar wind. The outflow is generally dense and slow wind results (Suess et al, 1996). Depending on the size of the heating source, the streamer will continue inflating until it has entirely evaporated, which has led us to call this process "streamer evaporation". For heat sources like those proposed to exist in the corona, the evaporation typically takes a few months. Of course this is not how the solar corona actually behaves - streamers can exist for several solar rotations. Evaporation is relevant for why there 
is a tendency for hot plasma to be released from streamers, but it does not explain why streamers reclose and sometimes last for several months. Nevertheless, the simulations of Steinolfson (1988) and Suess et al. (1996) clearly show the effect of evaporation in a polytropic gas simulation. This has led to the addition of thermal conduction to the models since this is a natural heat sink and will counteract evaporation. However, published simulations show this to only slow the evaporation rate (Suess et al, 1996).

These modeling results provide a strong reason for more comprehensive analysis of streamer energetics. Some of the important issues are the effects of more realistic descriptions of energy sinks, including radiative losses, and the effect of relaxing the assumption of axisymmetric flow used in the Suess et al (1996) simulation. Radiative losses may greatly reduce the rate of evaporation, while three-dimensionality increases the possibility that evaporation will be sporadic rather than continuous. Another important issue has to do with the physics of the volumetric heating because existing simulations all assume a source which is steady both in space and time. Realistically, coronal heating must depend on the ambient properties of the plasma and magnetic field, and introduction of an ambient-dependent source can also be expected to also lead to sporadic evaporation.

\section{SUMMARY}

The role of the magnetic field in modeling coronal expansion is receiving renewed emphasis under the stimulus of results from SOHO, Ulysses, and $\mathrm{YOHKOH}$. The importance of the plasma-magnetic field interaction is, to a great degree, summarized by the plasma $\beta$ and the empirical results have now placed limits on what this parameter is in coronal holes and streamers. Specifically, $\beta<<1$ in coronal holes out to at least $10 R_{S}$ and $\beta>1$ in streamers above $1.2 R_{\mathrm{s}}$. Knowing this leads directly to an explanation for the observed constancy of the radial IMF (as mapped to constant radius) and to a full understanding of the geometric spreading of coronal plumes and coronal holes. It poses, however, the question of how filamentary structures such as the plumes in coronal holes become mixed to produce the uniform flow seen further from the Sun.

That $\beta>1$ in streamers raises significant modeling and physical questions about the important processes in streamers and in the origin of slow solar wind which can only be answered with additional modeling, simulations, and observations. The definitive test will be whether a successful prediction is made for what the Solar Probe will encounter when it reaches the Sun.

\section{ACKNOWLEDGMENTS}

This work has been done with support from the Ulysses/SWOOPS (D.J. McComas, PI) and SOHO/UVCS (J. KohI, PI) instrument teams. In addition, I would like to acknowledge my extensive collaboration on this research with G. Poletto, S. Parhi, M. Sulkanen, A.-H. Wang, and S. T. Wu.

\section{REFERENCES}

Ahmad, I.A., \& G.L. Withbroe, EUV Analysis of Polar Plumes, Sol.Phys., 53, 397 (1977).

Billings, D.E., \& W.O.Roberts, The Origin of M-Region Geomagnetic Storms, Astrophys.Norv., 9, 147 (1964).

Chandrasekhar, S., "Hydrodynamic and Hydromagnetic Stability, Oxford University Press (1961).

Corti, G., G. Poletto, M. Romoli, J. Kohl, \& G. Noci, Physical Parameters in Plume and Interplume Regions from UVCS Observations, in "The Corona and Solar Wind Near Minimum Activity" (5th SOHO Workshop), ESA SP-404, p289 (1997).

Dahlburg, R.B., J.T. Karpen, G. Einaudi, \& P. Boncinelli, Acceleration of the Slow Solar Wind, in "Solar Jets \& Coronal Plumes" (Tan-Duc Guyenne, ed.), ESA SP-421, p199, European Space Agency, Noordwijk, The Netherlands (1998).

DeForest, C. E., J. T. Hoeksema, J. B. Gurman, B. J. Thompson, S. P. Plunkett, R. Howard, R. A. Harrison, \& D. M. Hassler, Polar Plume Anatomy: Results of a Co-ordinated Observation, Sol. Phys., 175, 393 (1998).

Feldman, W.C., J.R. Asbridge, S.J. Bame, E.E. Fenimore, \& J.T. Gosling. The Solar Origins of Solar Wind Interstream Flows: Near-Equatorial Coronal Streamers, J.Geophys.Res., 86, 5408 (1981).

Forsyth, R.J., A. Balogh, T.S. Horbury, G. Erdös, E.J. Smith, and M.E. Burton, The Heliospheric :Magnetic Field at Solar Minimum: Ulysses Observations from Pole to Pole, Astron. Astrophys, 316, 287 (1996). 
Gary, G.A., \& D. Alexander, Constructing the Coronal Magnetic Field, subm. to Sol. Phys. (1998).

Gosling, J.T., G. Borrini, J.R. Asbridge, S.J. Bame, W.C. Feldman, \& R.T. Hansen, Coronal Streamers in the Solar Wind at I AU, J.Geophys.Res., 86, 5438 (1981).

Grall, R. R., W. A. Coles, M. T. Klinglesmith, A. R. Breen, P. J. S. Williams, J. Markkanen, \& R. Esser, Rapid Acceleration of the Polar Solar Wind, Nature, 379. 429 (1996).

Habbal, S.R., R. Esser, M. Guhathakurta, \& R.R. Fisher, Flow Properties of the Solar Wind Derived from a Two-Fluid Model With Constraints from White Light and in situ Interplanetary Observations, Geophys. Res.Lett., 22, 1465 (1995).

Hardee, P.E., \& D.A. Clarke, Destabilization of Strongly Magnetized Jets, Astrophys.J., 449, 119 (1995).

Li, J., J.C. Raymond, L.W. Acton, J.L. Kohl, M. Romoli, G. Noci, \& G. Naletto, Physical Structure of a Coronal Streamer in the Closed Field Region Observed from UVCS/SOHO and SXT/YOHKOH, Astrophys.J., in press (1998).

Linker, J.A., \& Z. Mikic, Disruption of a Helmet Streamer by Photospheric Shear, Astrophys.J.Lett., 438, L45 (1995).

Mikic, Z., \& J. A. Linker, The Large-Scale Structure of the Solar Corona and Inner Heliosphere, in Solar Wind Eight. AIP Proc. 382 (D. Winterhalter et al., eds.), p104, Amer. Inst. Phys., Woodbury, N.W. (1996).

McComas, DJ., B.L. Barraclough, J.T. Gosling, C.M. Hammond, M. Neugebauer, A. Balogh, \& R.J. Forsyth, Structures in the Polar Solar Wind: Plasma and Field Observations from Ulysses, J.Geophys.Res., 100, 19,893 .

Neugebauer, M., and 12 other authors, The Spatial Structure of the Solar Wind and Comparisons with Solar Data and Models, J. Geophys. Res., in press (1998).

Noci, G., \& J.L. Kohl, and 23 others, The Quiescent Corona and Slow Solar Wind, in "The Corona and Solar Wind Near Minimum Activity" (A. Wilson, ed.), ESA SP-404, p75, European Space Agency, Noordwijk, The Netherlands (1997).

Poletto, G., S. Parenti, G. Noci, S. Livi, S.T. Suess, A. Balogh, \& D.J. McComas, Searching for Coronal Plumes in Ulysses Observations of the Far Solar Wind, Astron.Astrophys., 316, 374 (1996).

Phillips, J. L., S. J. Bame, A. Bames, B. L. Barraclough, W. C. Feldman, B. E. Goldstein, J. T. Gosling, G. W. Hoogeveen, D. J. McComas, M. Neugebauer, \& S. T. Suess, Ulysses Solar Wind Plasma Observations from Pole to Pole, Geophys. Res., Lett., 22, 3301 (1995).

Pneuman, G., \& R. Kopp, Gas-Magnetic Field Interactions in the Solar Corona, Sol.Phys., 18, 258 (1971).

Raymond, J., R. Suleiman, A. van Ballegooijen, \& J. Kohl, Absolute Abundances in Streamers from UVCS, in "Correlated Phenomena at the Sun, in the Heliosphere, and in Geospace" (B. Fleck, ed.), ESA SP-415, pxxx, European Space Agency, Noordwijk, The Netherlands (1997).

Schwadron, N.A., L.A. Fisk, \& T.H. Zurbuchen, On the Slow Solar Wind, Astrophys.J., in press (1998).

Sheeley, N.R., \& 18 others, Measurements of Flow Speeds in the Corona Between 1 and $30 \mathrm{R}_{\mathrm{s}}$, Astrophys.J., 484, 472 (1997).

Steinolfson, R.S., Density and White Light Brightness in Looplike Coronal Mass Ejections: Importance of the Preevent Atmosphere, J.Geophys. Res., 93, 14,261 (1988).

Steinolfson, R.S., S.T. Suess, \& S.T. Wu, The Steady Global Corona, Ap.J., 255, 730 (1982).

Steinolfson, R.S., \& A.J. Hundhausen, Density and White-Light Brightness in Looplike Coronal Transients: Transient Simulations, J.Geophys.Res., 93, 14,268 (1988).

Suess,S.T., The Relationship Between Coronal \& Interplanetary Magnetic Fields, Adv.Spa.Res., 13, 31 (1993).

Suess, S.T., Models of Plumes: Their Flow, Their Geometric Spreading, and Their Mixing with Interplume Flow, in "Solar Jets \& Coronal Plumes" (Tan-Duc Guyenne, ed.), ESA SP-421, p223, European Space Agency, Noordwijk, The Netherlands (1998).

Suess, S.T., A.K. Richter, C.R. Winge, Jr., and S.F. Nerney, Solar Polar Coronal Hole - A Mathematical Simulation, Astrophys. J., 217, 296 (1977).

Suess, S.T., \& E. Smith, Latitudinal Dependence of the Radial IMF Component: Coronal Imprint, Geophys. Res. Lett., 23(22), 3267 (1996).

Suess, S.T., E. Smith, J. Phillips, B.E. Goldstein, \& S. Nemey, Latitudinal Dependence of the Radial IMF Component: Interplanetary Imprint, Astron. Astrophys, 316, 304 (1996).

Suess, S.T., A.-H. Wang, \& S.T. Wu, Volumetric Heating in Coronal Streamers, J. Geophys. Res., 101, 19,957 (1996).

Suess, S.T., G. Poletto, A.-H. Wang, S.T. Wu, \& I. Cuseri, The Geometric Spreading of Coronal Plumes and Coronal Holes, Sol. Phys., in press (1998).

Wang, A.-H., S.T. Wu, S.T. Suess, \& G. Poletto, Numerical Modeling of Coronal Mass Ejections Based on Various Preevent Model Atmospheres, Sol.Phys., 161, 365 (1995). 
Wang, A.-H., S.T. Wu, S.T. Suess, \& G. Poletto, Global Model of the Corona With Heat and Momentum Addition, J. Geophys. Res., 103, 1913 (1998).

Wang, Y.-M., On the Latitude and Solar Cycle Dependence of the Interplanetary Magnetic Field Strength, $J$. Geophys. Res., 98, 3529 (1993).

Wu, S.T., W.P. Guo, \& M. Dryer, Dynamical Evolution of a Coronal Streamer - Flux Rope System. II. A SelfConsistent Non-Planar Magnetohydrodynamic Simulation, Sol.Phys., 170, 265 (1997). 